\title{
Wissen wir, was ein Körper kann?
}

ARNO BÖHLER

\section{Von der falschen Herrschaft des Geistes über den Körper}

Wenn wir einen Blick in die Geschichte der Philosophie werfen, fällt auf, dass Philosophen immer wieder darüber nachgedacht haben, wie Körper geistig gezügelt, gesellschaftlich diszipliniert und willentlich auf bestimmte ideelle Ziele hin ausgerichtet werden können.

Für Spinoza gründet dieses Verhalten im klassischen Bild des Denkens, das davon ausgegangen ist, dass Körper völlig inaktiv wären, wenn sie vom Geist nicht bewegt würden. So fest waren sie davon überzeugt, „dass der Körper bloß auf Geheiß des Geistes bald sich bewegt, bald ruht und dass er sehr vieles verrichtet, was allein von dem Willen des Geistes und dessen Erfindungskunst abhängt.“ (Spinoza 2007 [3p2s]: 229)

Durch das Vorurteil, dass die Ursachen von körperlichen Bewegungen allein in geistigen Entschlüssen eines „Subjekts“ begründet wären, haben sie sich nie ernsthaft gefragt, ob es nicht eine Eigenaktivität der Körper gibt, in der sie von sich aus (materiell) aktiv werden, ohne von einem Geist bewegt zu werden. Im Überspringen dieser Frage ortet Spinoza eine entscheidende Forschungslücke der Philosophie. Denn: „was der Körper kann, hat bislang noch niemand bestimmt; d. h. die Erfahrung hat bislang niemanden darüber belehrt, was der Körper bloß nach Gesetzen der Natur, insofern diese allein als körperlich angesehen wird, verrichten kann und was allein dadurch, dass er von dem Geist bestimmt wird." (Spinoza 2007 [3p2s]: 229)

Was der Geist, was der Wille, was Bewusstsein und Selbstbewusstsein zu leisten vermögen, das haben viele Philosophen untersucht. Aber zu fragen, was ein Körper kann, insofern er bloß den Gesetzen seiner Physis folgt, das hat noch niemand philosophisch zu bestimmen versucht. Und das, obwohl es eine Reihe von Phänomenen gibt, in denen uns die Erfahrung lehrt, das Körper sehr wohl 
aktiv werden können, ohne dass ihre Aktivität von uns willentlich verursacht oder geistig kontrolliert würde.

Als Beispiel führt Spinoza gerne die Körper von Nachtwandlern an, die im Schlaf vieles tun, was sie sich im wachen Zustand nicht zutrauen würden (vgl. Spinoza 2007 [3p2s]: 229, 23I). Auch die außerordentlichen Leistungen niederer Lebewesen, die in ihrem Dasein Erstaunliches zu Wege bringen, obwohl sie über keinen strategisch planenden Geist verfügen, weisen für ihn in die Richtung, dass Körper ohne mentale Kontrolle unterschwellig handeln und vieles können, worüber der Geist nur staunen kann (vgl. Spinoza 2007 [3p2s]: 229). Vor allem ist es aber die komplexe Fabrikation des menschlichen Körpers [corporis fabricam], den die Natur im Zuge ihres ewigen Ausdruckgeschehens zunächst einmal schlafwandlerisch konzipiert hat, der Spinoza wiederholt auf die Frage zurückkommen lässt: Wissen wir, was ein Körper kann? „Denn bislang kennt niemand den Bau des Körpers so genau, dass er alle seine Funktionen erklären könnte.“ (Spinoza 2007 [3p2s]: 229)

Diese und andere Phänomene deuten eindeutig daraufhin, dass es eine dunkle, nachtaktive Zone in der Natur geben muss, in der Körper von sich aus auf höchst intelligente Art und Weise auf ihre Umgebung reagieren, ohne dass diese Operationen mental gesteuert, geistig geplant oder gar willentlich verursacht worden wären.

\section{Spinozas Lehre vom Parallelismus der beiden Attribute Denken und Ausdehnung}

Wollen wir uns von dem falschen Bild des Denkens lösen, das den Handlungscharakter von Körpern übersieht, dann müssen wir mit Spinoza einsehen, „dass der Geist und der Körper ein und dasselbe Ding sind, das bald unter dem Attribut Denken, bald unter dem Attribut Ausdehnung begriffen wird." (Spinoza 2007 [3p2s]: 227) Denn erst dann, wenn Körper und Geist als unterschiedliche Attribute ein und derselben Substanz erfasst werden, wird die Vorherrschaft des Geistes über den Körper vermieden; ebenso wie die Vorherrschaft des Körpers über den Geist. Führt jede Steigerung des Tätigkeitsvermögens in einem der beiden Attribute gemäß der Lehre des Parallelismus doch auch zur Steigerung des Tätigkeitsvermögens im anderen Attribut: Wo immer der Vermögensgrad unserer Physis erhöht wird, wird auch unsere Denkkraft erhöht und umgekehrt. Die praktische Bedeutung dieser Lehre besteht offenkundig im 
Außer-Kraft-Setzen des klassischen Bilds des Denkens, das die geistige Zügelung und Beherrschung unserer Begierden forderte. Denn:

„[...] wenn der Körper handelte, litt die Seele, sagte man, und die Seele handelte nicht, ohne dass der Körper seinerseits litt (Regel des umgekehrten Verhältnisses, vgl. Descartes, Abhandlungen über die Leidenschaften, Artikel I und 2). Der Ethik zufolge ist demgegenüber das, was in der Seele Tätigkeit (action) darstellt, notwendigerweise auch im Körper Tätigkeit; das, was im Körper Leidenschaft (passion) ist, stellt notwendigerweise auch in der Seele Leidenschaft dar.“ (Deleuze I988: 28)

Besonders deutlich zeigt sich die Gültigkeit des Parallelismus von Geist und Materie für Spinoza am Phänomen des Schlafs. Lässt sich doch nicht bestreiten, dass der Geist unfähig zum Denken wird, sobald der Körper seine Tagesaktivität einstellt. „Denn wenn der Körper im Schlaf ruht, bleibt zugleich der Geist im Schlummer und hat, anders als im wachen Zustand, nicht die Fähigkeit zu planen und zu erfinden.“ (Spinoza 2007 [3p2s]: 23I). Besonders im Tiefschlaf zeigt sich, dass im Zustand vollkommener körperlicher Ruhe auch die geistigen Aktivitäten des Körpers gänzlich zur Ruhe kommen; - was die Wahrheit des Lehrsatzes unumwunden belegt, dass jeder Reduktion einer Aktivität im Attribut Ausdehnung eine entsprechende Reduktion der Aktivität im Attribut Denken entspricht und umgekehrt (vgl. Spinoza 2007 [2p7]: I09; [3p2s]: 227229; [3ріг]: 243-247).

Aber lehrt uns das Phänomen Tiefschlaf nicht auch, dass die Eigenaktivität unserer Körper die Aktivität unseres Selbstbewusstseins Nacht für Nacht überdauert? Sind unsere Körper nicht auch dann noch nacht-aktiv tätig, wenn wir uns im Tiefschlaf befinden und uns nichts mehr vorstellen? Weder die Welt um uns herum noch uns selbst? (Vgl. Böhler/Granzer 20I4) Denn wenn wir in der Tiefschlafphase sogar für das Träumen zu müde geworden sind (vgl. Nietzsche GM KSA 5: 38I), bleibt die große Vernunft unseres Leibes weiterhin damit beschäftigt, uns am Sein zu erhalten, indem sie sich abseits von jeglicher mentalen Kontrolle um die vitale Aufrechterhaltung unserer lebenswichtigen Funktionen sorgt: um das Intakthalten unserer Atmung, unserer Verdauung, Blutzirkulation, um ein funktionierendes Herzkreislaufsystem und vieles mehr.

Sollte dieser Sachverhalt Philosophen nicht zu denken geben? - Dass sie ohne die Nachtaktivität der großen Vernunft unseres Leibes nicht eine einzige Nacht als denkende Subjekte überleben würden? Und wird dieser paradoxe Sachverhalt nicht noch erstaunlicher, wenn wir bedenken, dass der Tiefschlaf in der Regel ein Zustand elementarer Regeneration und Körperentspannung 
darstellt? - Kein bloßer Mangel an Bewusstheit, sondern ein schlafwandlerisch sicheres Operieren unserer Körper, in dem sie in der Tat auf beeindruckende Art und Weise ihr Know-How demonstrieren, unsere Physis ganz ohne mentale Kontrolle selbstaktiv regenerieren zu können? Fast so, als ob sie die nächtliche Absenz unserer selbstbewussten mentalen Fähigkeiten geradezu herbeisehnen würden, um die Gunst dieser mitternächtlichen Geisterstunde für die physische Regeneration unseres leiblichen In-der-Welt-Seins zu nutzen.

Dass dieser paradoxe Zustand - physisch da, mental absent -, der uns vornehmlich gegen Mitternacht heimsucht, von vielen Mythen und Geisterlegenden umwoben ist, wundert nicht (vgl. Derrida 1995: 27). Markiert er offensichtlich doch jene Unzeit des Denkens, von der Nietzsche am Ende von Also sprach Zarathustra sagt, sie sei elementar tiefer als der Tag gedacht.

Aber wenn es auch evident ist, dass die nachtwandlerische Performance unserer Körper im Tiefschlaf ohne ein Subjekt auskommt, das sie mental steuert und bewusst kontrolliert, so antizipiert Spinoza doch, dass sich seine Gegner mit solchen Beispielen, die uns ein Unbewusstes des Denkens zu denken nötigen, noch nicht zufrieden geben werden. Ahnt er im Vorhinein doch schon ihre Einwände:

„Auch werden sie wohl sagen, allein den Gesetzen der Natur, insofern sie nur als körperlich angesehen wird, könnten nicht die Ursachen von Gebäuden, Gemälden und anderen Dingen dieser Art, die allein aus menschlicher Kunst entstehen, entnommen werden, und der menschliche Körper wäre doch nie imstande, eine Kirche zu erbauen, ohne dazu von dem Geist bestimmt und angeleitet zu werden." (Spinoza 2007 [3p2s]: 23I)

Aber auch auf diesen Einwand antwortet Spinoza wieder mit dem uns inzwischen schon vertrauten Argument:

„Allein ich habe schon darauf hingewiesen, dass sie gar nicht wissen, was der Körper kann oder was sich aus der Betrachtung allein seiner Natur herleiten lässt, ja dass sie selbst erfahren, dass allein nach den Gesetzen der Natur vieles geschieht, von dem sie niemals geglaubt hätten, es könne ohne Anleitung des Geistes geschehen, z. B. was Nachtwandler im Schlaf tun, worüber sie sich dann im wachen Zustand wundern." (Spinoza 2007 [3p2s]: 23I) 


\section{Das subjektlose Begehren des conatus: Spinozas ethischer Immoralismus}

Gilles Deleuze hat in seinen beiden Monographien zu Spinoza besonders darauf Wert gelegt, dass Spinoza seine Lehre von der nachtwandlerischen Produktivität der Körper beinahe als Kriegserklärung verstanden wissen wollte.

„Wenn Spinoza sagt, wir wissen nicht einmal, was ein Körper kann, dann ist diese Formulierung fast ein Schlachtruf [...]“ (Deleuze I993: 225);

„[...] wir sprechen vom Bewusstsein und seinen Beschlüssen, vom Willen und seinen Wirkungen, von tausend Mitteln, den Körper zu bewegen, den Körper und die Leidenschaften zu beherrschen - aber wir wissen nicht einmal, was der Körper alles vermag." (Deleuze i988: 27)

Durch die Art und Weise, wie Spinoza diese Frage seinen Gegnern an den Kopf wirft und im Gesamtkontext seiner Ethik inszeniert, ist klar, dass ihn diese Frage selbst emotional bewegt. Sie freizulegen ist für ihn nicht nur Programm einer erst im Kommen begriffenen Philosophie passiver Synthesis (vgl. Böhler 2005; Deleuze I997: 99-I30; Heidegger I99I; Husserl I966; Rölli 2003 sowie Damasio 2009: 243-253), sie ist offenkundig auch ein „persönliches“ Anliegen von ihm, das er selbst libidinös besetzt hat. - Gerade so, als ob es sich bei dieser Forschungsfrage insgeheim um das Herzstück seiner ganzen Ethik handeln würde, an der ihm selbst wesentlich gelegen ist; - gerade so, als ob sich das dominante Begehren seines eigenen conatus gerade hier ausdrücken und zur Sprache bringen möchte.

Auf jeden Fall deckt diese Frage für Spinoza eine entscheidende Blöße der traditionellen Philosophie auf. Eine Forschungslücke, die ihr subversives Potential erst dort in vollem Ausmaß enthüllt, wo die Lehre von der prä-subjektiven Handlungspotenz der Körper nicht nur als naturphilosophische Theorie, sondern als ethisch relevantes Problem aufgefasst wird. Denn erst mit diesem Schritt realisiert er jene ethische Umwertung aller bisherigen moralischen Werte, die Nietzsche im I9. Jhd. wiederholt, indem sie beide ihr Denken der Körper mit praktischen Handlungstheorien verknüpften: „Hier liegen die drei großen Ähnlichkeiten mit Nietzsche. Schon zu seinen Lebzeiten bezichtigte man Spinoza deswegen des Materialismus, der Immoralität und des Atheismus." (Deleuze I988: 27) 
Nicht unsere fehlende Einsicht in die adäquaten Ursachen physischer Prozesse, sondern die postulierte Unwissenheit gegenüber den adäquaten Ursachen unserer moralischen Handlungen ist es, die an Spinoza massenhaft empörte: „[...] ihr wisst nicht, wozu ihr im Guten wie im Schlechten fähig seid, ihr wisst nicht im Voraus, was ein Körper oder eine Seele in solcher Begegnung, jener Anordnung, jener Kombination vermag." (Deleuze 1988: 162)

Die physiologische Unwissenheitserklärung ist eine Provokation, die ethische aber ein Skandal. Reduziert sie Freiheitssubjekte, und damit auch Fragen der Sittlichkeit und Moral, doch auf dunkle Triebtätigkeiten, die unser bewusstes Denken insgeheim dirigieren.

„So glaubt das kleine Kind, es verlange frei nach der Milch, der zornige Junge, er wolle Rache, und der ängstliche Mensch, er wolle die Flucht. So glaubt der Trunkene, er rede aus freier Entscheidung des Geistes, was er nachher, wieder nüchtern, lieber verschwiegen haben wollte. So glauben der Faselhans, das Plappermaul, der Kindskopf und viele Leute dieses Schlages aus freier Entscheidung des Geistes zu reden, während sie doch bloß ihrem Rededrang, den sie nun einmal haben, nicht widerstehen können, so dass gerade die Erfahrung nicht weniger klar als die Vernunft lehrt, dass Menschen sich allein deshalb für frei halten, weil sie sich ihrer Handlungen bewusst sind, aber die Ursachen nicht kennen, von denen sie bestimmt werden. Außerdem, dass die Entscheidungen des Geistes nichts sind als die Triebe selbst, die entsprechend der verschiedenen Disposition des Körpers verschiedenartig sind.“ (Spinoza 2007 [3p2s]: 233; vgl. auch ebd.: 235-237)

Deutlicher kann man nicht ausdrücken, dass das moralische Freiheitsproblem eine Mär darstellt, die ein fiktives, intelligibles Subjekt konstruiert, das, wie Nietzsche womöglich sagen würde, nirgendwo anders als in die Welt „imaginärer Ursachen“ (Nietzsche GD KSA 6: 92) gehört. Für beide fungiert die Fabel freiheitlicher Subjekte vielmehr als schädlicher Freiheitswahn, der uns in Wahrheit gerade davon abhält, die wirklichen Ursachen unseres Handelns adäquat einzusehen und damit wirklich frei zu werden. Menschen, die sich von ihm nicht befreit haben, werden von wirklicher Freiheit daher wohl auch in Zukunft weiterhin nur mit offenen Augen träumen können.

„Denn wenn sie sagen, menschliche Handlungen hingen von dem Willen ab, dann sind das Worte, für die sie keine Idee haben. Was nämlich der Wille ist und wie er den Körper bewegt, das wissen sie alle nicht [...]" (Spinoza 2007 [2p35s]: I7I). 
Wieder stoßen wir auf dieselbe Denkfigur. Das, was den Körper bewegt, ist weder ein körperloser Geist noch ein intelligibler Freiheitswille, sondern ein allen Körpern immanentes Streben (vgl. Cook 2006: I55-I56), das jeden von ihnen motiviert, sich in seinem Da-sein am Sein zu erhalten.

„Bezieht sich dieses Streben allein auf den Geist, wird es Wille [voluntas] genannt, bezieht es sich aber auf den Geist und zugleich auf den Körper, Trieb [appetitus]. Er, der Trieb, ist somit nichts anderes als genau die Essenz des Menschen, aus dessen Natur das, was der eigenen Erhaltung dient, notwendigerweise folgt, mithin ist der Mensch bestimmt, es zu tun.“ (Spinoza 2007 [3p9s]: 243)

Anstatt den Versuch zu machen, die Handlungen von Subjekten aus der selbstimmanenten Seinsaktivität ihres conatus heraus $\mathrm{zu}$ verstehen, der ein jedes Ding so in sich selbst sein lässt, dass es von sich aus danach strebt, sich am Sein zu erhalten, werden Handlungen von Subjekten moralisch als Äußerungen eines freien Willens missdeutet. Wodurch man sich immer tiefer in den Wahn „freiheitlicher Subjekte“ verstrickt (vgl. Matheron 2006: 297-307). Denn so, wie die Sonne ihre Strahlen automatisch in völligem Einklang mit den physikalischen Gesetzen ihrer Natur (physio-logisch korrekt) aussendet, ohne dass ihr ein Geist extra befehlen müsste, dies zu tun; gerade so bringt die schaffende Natur für Spinoza die erschaffene Natur (vgl. Spinoza 2007 [Ip29-Ip3I]: 63-67) immer schon als geometrische Folge eines ewigen, selbstimmanenten Ausdrucksgeschehens derselben hervor. - Im Zuge eines Ereignisses also, kraft dessen die ewigen Attribute der schaffenden Natur (natura naturans) in der erschaffenen Natur (natura naturata) modal ausgedrückt werden und damit (zeiträumlich) zum Vorschein kommen.

So wenig sich die Sonne dazu entschließen muss, zu scheinen, um zu scheinen, so wenig geht dem ewigen Ausdrucksgeschehen der Natur im Spinozismus also ein geistiger Entschluss eines (göttlichen) Subjekts voraus, die Welt erschaffen zu wollen, damit sie aus seiner Entschlusskraft in der Tat überhaupt hervorgehe. Alles geschieht für Spinoza vielmehr aus einer inneren Notwendigkeit der schaffenden Natur selbst. Oder, wie er im Lehrsatz 29 des ersten Buchs seiner Ethik schreibt: „Demnach ist alles aus der Notwendigkeit der göttlichen Natur bestimmt, nicht nur überhaupt zu existieren, sondern auch in einer bestimmten Weise zu existieren und etwas zu bewirken; und es gibt nichts Zufälliges.“ (Spinoza 2007 [Ip29d]: 63)

Wie es der All-Einen Substanz nie frei stand, nicht zu sein, da sie nicht aus etwas anderem allererst geworden ist, sondern sich seit jeher in sich selbst und 
durch sich selbst konzipiert und erfasst (vgl. Spinoza 2007 [Id3]: 5), so steht es endlichen Modi nicht mehr frei, nicht zu sein, sobald sie angefangen haben zu sein. Denn sobald ein endlicher Modus inmitten der Welt existent geworden ist, existiert auch er notwendigerweise gerade so, wie er existiert: mit einer bestimmten Wirkfähigkeit, einer konkreten Potenz, einem diskreten Optimum an Kraft, das ihm kraft seiner Geburt mitgegeben wird und daher von nun an per se zukommt.

Aber nicht nur Spinoza, auch Nietzsche hat uns wiederholt auf die Gefahr aufmerksam gemacht, dass uns die Grammatik von Sätzen wie „Die Sonne scheint“ verführt $z u$ glauben, es gäbe unabhängig vom Prädikat "scheinen“ ein davon losgelöstes Subjekt "Sonne“, dem es freistünde, zu scheinen oder nicht zu scheinen. Für beide Denker handelt es sich bei solchen Propositionen letztlich um ein moralisch motiviertes Missverständnis, das den Satzbau vieler Sprachen inzwischen entscheidend prägt, sodass er jene, die eine so geartete Sprache regelkonform gebrauchen, unweigerlich dazu verleitet, inadäquate Ideen von der Welt zu bilden. D. h. illusionäre Vorstellungen wie eben die, es gäbe hinter dem Scheinen der Sonne oder hinter dem Blitzen eines Blitzes noch einen Täter namens Sonne bzw. Blitz, der für das Scheinen oder Blitzen verantwortlich zeichnen würde.

„Ebenso nämlich wie das Volk den Blitz von seinem Leuchten trennt und letzteres als Thun, als Wirkung eines Subjekts nimmt, das Blitz heißt, so trennt die Volks-Moral auch die Stärke von den Äußerungen der Stärke ab, wie als ob es hinter dem Starken ein indifferentes Substrat gäbe, dem es freistünde, Stärke zu äußern oder auch nicht. Aber es giebt kein solches Substrat; es giebt kein ,Sein' hinter dem Thun, Wirken, Werden; ,der Thäter' ist zum Thun bloß hinzugedichtet, - das Thun ist alles." (Nietzsche GM KSA 5: 279. Vgl. auch Nietzsche GD KSA 6: 77, 9I)

Daraus folgt, und damit kommen wir erst zum wunden Punkt einer solchen Doktrin subjektloser Tätigkeiten zurück,

„[...] dass Menschen, die sagen, diese oder jene Tätigkeit des Körpers rühre von einem Geist her, der die Herrschaft über den Körper habe, nicht wissen, was sie sagen, und nichts anderes tun, als mit hochtrabenden Worten einzugestehen, dass sie die wahre Ursache einer solchen Tätigkeit nicht kennen und an ihr auch nicht interessiert sind.“ (Spinoza 2007 [3p2s]: 229) 
Wagen wir im Folgenden also den Versuch, gerade hier, wo Philosophen traditionell nicht mehr zu zweifeln wagen, unser großes Fragezeichen zu setzen: Ob man nämlich nicht gerade hier, wie Nietzsche in Jenseits von Gut und Böse schreibt, umlernen und einsehen muss, dass unser Bewusstsein unseren Affekten und Instinkten gerade nicht entgegengesetzt ist, wie bisher meistens vermutet wurde, weil man den größten Teil des bewussten Denkens noch zu den Instinkttätigkeiten rechnen muss, „sogar im Falle des philosophischen Denkens; [...]“ (Nietzsche JGB KSA 5: I7).

\section{Asketisches Begehren}

Zu lange, so könnte man mit Spinoza und Nietzsche sagen, haben Philosophen bislang verabsäumt $\mathrm{zu}$ fragen, was der Appetit strebender Körper von sich aus alles zu leisten vermag. $\mathrm{Zu}$ lange haben sie sich auf die Analyse geistig-mentaler Tätigkeiten fokussiert und dabei die große Vernunft des Leibes aus den Augen verloren, in der die Natur schlafwandlerisch operiert.

Dass Philosophen, bekanntlich waren sie bisher fast ausschließlich männlichen Geschlechts, die Frage nach den dunklen Zonen der Körper kaum gestellt haben, geschieht, zumindest für Nietzsche, nicht rein zufällig. Hat man ihnen nämlich erst einmal lange genug zwischen die Zeilen und auf die Finger geschaut (vgl. Nietzsche JGB KSA 5: I7), kommt man unweigerlich zu der Einsicht, dass dieses Versäumnis mit ihrer asketischen Begehrensstruktur zusammenhängt. Irgendetwas, das ihre Leiblichkeit durchherrscht, irgendein tiefeingefleischter Instinkt hat ihnen kategorisch befohlen, der Frage nach dem Eigensinn von Körpern auszuweichen, um an die Stelle begehrlicher Körper ein freies, von der Sinnlichkeit unberührtes intelligibles Subjekt zu setzen. Offenkundig scheint ihnen ein solches näher zu liegen als das empirische Subjekt, auf das sie in ihrer eigenen Leibes-Tiefe stoßen. - Zumindest insgeheim; denn hier nicht zum Bewusstsein zu kommen, gehört ganz entscheidend zur Struktur ihres Begehrens; - zu jener Strebensart also, die sie grosso modo dahin treibt, nach übersinnlichen Ideen und einer intelligiblen Freiheit zu suchen, um die Blöße ihres empirischen Charakters zu verdecken; lässt sich der von Nietzsche erstmals klar eingesehene historische Tatbestand doch kaum leugnen:

„Es besteht unbestreitbar, so lange es Philosophen auf Erden giebt und überall, wo es Philosophen gegeben hat (von Indien bis England, um die entgegengesetzten Pole der Begabung für Philosophie zu nehmen) eine eigentliche Philosophen-Gereiztheit und 
-Rancune gegen die Sinnlichkeit - Schopenhauer ist nur deren beredtester und, wenn man das Ohr dafür hat, auch hinreissendster und entzückendster Ausbruch -;" (Nietzsche GM KSA 5: 350).

Nicht der Wille zur Wahrheit, sondern der Widerwille gegen die Sinnlichkeit ist für Nietzsche also der entscheidende Beweggrund, der klassische Philosophen nach intelligiblen Ursachen, übersinnlichen Ideen und einer interesselosen Anschauung des Lebens Ausschau halten ließ. Sie taten dies nicht aus selbstlosen Beweggründen, sondern „aus dem allerstärksten, allerpersönlichsten Interesse: dem des Torturirten, der von seiner Tortur loskommt“ (Nietzsche GM KSA 5: 349). In der Regel wollten sie alle nur eines: „Ruhe in allen Souterrains;“ (Nietzsche GM KSA 5: 352). Und fehlte das asketische Begehrensmoment an einem Philosophen, dann konnte man sicher gehen, dass er nur ein sogenannter „Philosoph“ war. Ein Philosoph unter Anführungszeichen, dem Entscheidendes fehlte, um ein echter, wahrer, ernst zu nehmender Philosoph genannt zu werden (vgl. Nietzsche GM KSA 5: 350).

Wenn wir uns also fragen, was es bedeutet, dass Philosophen bislang dem asketischen Ideal huldigten, so bekommen wir für Nietzsche an dieser Stelle einen ersten Wink: Philosophen wollten durch die Bejahung asketischer Ideale von der Tortur der Sinnlichkeit loskommen. Sie brauchten die Konstruktion imaginärer Ursachen, um die Blöße ihrer Leibes-Tiefe zu verdecken. Nannte die Metaphysik diesen Akt der Verstellung bislang nicht Wahrheit?

\section{Der Asket: Arthur Schopenhauer}

In der neueren Philosophiegeschichte, wir haben es soeben gehört, findet Nietzsche das ehrwürdigste Beispiel einer solchen asketischen Denkfigur im Denken Arthur Schopenhauers. Wenn Schopenhauer die Erfahrung des Schönen als eine ästhetische Empfindung beschreibt, die „der geschlechtlichen ,Interessiertheit" entgegenwirke“ (Nietzsche GM KSA 5: 348), dann bringt er ganz unverblümt den äußersten Glückszustand zur Sprache, den er selbst am eigenen Leib zu empfinden im Stande war. Ganz im Gegensatz etwa zu Stendhal, der die entscheidende Wirkung des Schönen auf unseren Appetit gerade in der "Erregung des Willens (,des Interesses“) durch das Schöne [erkennt]“ (Nietzsche GM KSA 5: 349). Ganz im Sinne Nietzsches deutet er das Schöne also als Stimulanz des Lebens - „das Schöne verspricht Glück“ (Nietzsche GM KSA 5: 349) -, während Schopenhauer in seiner höchsten ästhetischen Empfindung gerade 
die Erlösung vom schnöden Willensdrang des Lebens feiert, wie er selbst sagt, wenn er schreibt:

"[d]as ist der schmerzlose Zustand, den Epikur als das höchste Gut und als den Zustand der Götter pries; wir sind, für jenen Augenblick, des schnöden Willensdrangs entledigt, wir feiern den Sabbat der Zuchthausarbeit des Wollens, das Rad des Ixion steht still.“ (Zit. nach Nietzsche GM KSA 5: 348; vgl. Schopenhauer WWV I 23I)

Nicht das aktive, schaffende „frei wozu“ (Nietzsche Z KSA 4: 8I) ist bei Schopenhauer also das treibende Element des Schönen in Kunst und Natur, sondern ein den Lebenswillen hemmender, zurückdrängender Affekt, der uns vom Schmerz des Lebens befreit und damit von der Knechtschaft des Willens erlöst, die uns an das Feuerrad des Lebens bindet. Schopenhauers Schilderungen des eigenen ästhetischen Empfindens sind für Nietzsche besonders lehrreich, weil er gerade in ästhetischen Belangen völlig instinktsicher argumentiert. Die kontemplative Wirkung des Schönen auf seine Physis war ihm oberste sinnliche Gewissheit; d. h. eine Erfahrung, die ihn ohne Zweifel am eigenen Leib regelmäßig heimsuchte und bis in die äußersten Enden seiner Leiblichkeit hinein entzückte (vgl. Nietzsche GM KSA 5: 347-349).

Und doch schleicht sich für Nietzsche gerade hier, in der Selbstinterpretation seines eigenen ästhetischen Empfindens, bei Schopenhauer ein gravierendes Missverständnis gegenüber dem Leben ein. Und zwar genau dort, wo er die kontemplative Wirkung des Schönen auf sein eigenes Gemüt als Akt der Verneinung des Wollens, sprich der Lebensverneinung deutet. Bei dieser Interpretation handelt es sich für Nietzsche um eine glatte Verkehrung der wirklichen Tatbestände. Was Schopenhauer in solchen Glücksmomenten erlebte, war gerade nicht die Verneinung der Zuchthausarbeit des Wollens, wie er selbst sagt, sondern das Aufblitzen einer aisthetischen Perspektive auf das Leben, die es ihm plötzlich erlaubte, das Leben insgesamt wieder zu bejahen und begehrenswert zu finden. Nicht die Negation des schnöden Willensdrangs, sondern der Wille zu seinem Optimum an Lebendigkeit war es, der ihn nach asketischen Lebensformen und Denkfiguren streben ließ. Denn: „Jedes Thier, somit auch la bête philosophe, strebt instinktiv nach einem Optimum von günstigen Bedingungen, unter denen es seine Kraft ganz herauslassen kann und sein Maximum im Machtgefühl erreicht; [...]“ (Nietzsche GM KSA 5: 350).

Wenn wir uns also fragen, was einen Philosophen klassischerweise dazu trieb, asketische Ideale zu bejahen, dann lautet die Antwort von Nietzsche nicht nur, dass er damit einfach von der Tortur seiner eigenen Sinnlichkeit und Lei- 
bes-Tiefe loskommen wollte. Lächelte er beim Anblick derselben offenkundig doch auch „einem Optimum der Bedingungen höchster und kühnster Geistigkeit zu, - er verneint nicht damit, das Dasein', er bejaht darin vielmehr sein Dasein und nur sein Dasein“ (Nietzsche GM KSA 5, 351).

Nicht die Tugendhaftigkeit oder gar Selbstlosigkeit der Philosophen ist es, die sie beispielsweise nach rationalen Vernunftgründen streben lässt, sondern der Appetit ihrer Körper, insofern er sich auf der Suche nach seinem Optimum an Kraft, an Macht, an „agendi potentia“ (Spinoza 2007 [3praef]: 220) inmitten der Welt befindet. Er ließ Schopenhauer insgeheim nach asketischen Idealen streben, Platon nach übersinnlichen Ideen Ausschau halten oder Kant ein intelligibles Freiheitssubjekt postulieren. - Allesamt libidinös gefärbte Weltentwürfe, in denen nicht nur festgeschrieben wird, was uns das Erstrebenswerteste überhaupt heißen sollte, sondern auch definiert wird, was der Erreichung unseres Optimums entgegenläuft, das wir alle als höchstes Glück erstreben sollten; denn: „jedes Thier perhorresziert ebenso instinktiv und mit einer Witterung, die ,höher als die Vernunft', alle Art Störenfriede und Hindernisse, die sich ihm über diesen Weg zum Optimum legen oder legen könnten [...]“ (Nietzsche GM KSA 5: 350).

Erst dort, wo die rationale Maskierung der Partikularität des eigenen Begehrens gelingt, indem ihr massenhaft geglaubt wird, kann das eigene Begehren eine normative Funktion übernehmen und damit den hegemonialen Anspruch stellen, die maßgebliche Form von Begehren schlechthin zu sein. In Kontingenz, Hegemonie, Universalität haben Butler, Žižek und Laclau die Rhetorizität dieses Verfahrens schonungslos aufgedeckt. „Damit es Hegemonie gibt, ist es nötig, dass die partiellen Ziele einer Gruppe als Name für eine Universalität fungieren, die jene transzendiert - das ist die für die hegemoniale Verbindung konstitutive Synekdoche." (Laclau 20I3: 73-74)

Nur unter dem Schutz-Schleier des rhetorischen Verfahrens, in dem ein Modi des Begehrens die Funktion übernehmen konnte, die adäquate Form von Begehren überhaupt zu repräsentieren, konnte demnach die klassisch asketische Form des Begehrens den normativen Anspruch erheben, das echte Begehren jedes wahren Philosophen überhaupt zu sein. Solange dieser Anspruch von der Sittlichkeit der Sitte unbefragt als konstitutiver Bestandteil des klassischen Anforderungsprofil an Philosophen angesehen wurde, hatte diese Synekdoche eben auch die Macht, Kraft und Autorität, maßgeblich darüber zu entscheiden, ob es sich bei einem Bewerber der Philosophie um einen qualifizierten Bewerber handelt, oder bloß um einen sogenannten „Philosophen“ unter Anführungszeichen, dem es an der „rechten“ Art des Begehrens mangelt. 


\section{Der asketische Priester}

Soll das ganze Ausmaß der philosophischen Relevanz ermessen werden, die mit dem Problem asketischer Ideale zusammenhängt, dann genügt es für Nietzsche nicht, diese Frage nur auf die asketische Begehrensstruktur klassischer Philosophen und Wissenschafter ${ }^{1}$ zu beziehen. Vielmehr muss man die Figur des asketischen Priesters in Analyse schicken, wenn man die Bedeutung dieser Frage für unser aller Leben wirklich verstehen möchte. Denn erst mit dieser paradoxen Lebensform, in der sich ein Lebewesen gegen das Leben insgesamt wendet, „rücken wir dem Problem: was bedeutet das asketische Ideal? ernsthaft auf den Leib.“ (Nietzsche GM KSA 5: 36I) Wird uns in diesem Fall doch „das physiologische Ringen eines Menschen mit dem Tod“ vor Augen geführt: „(genauer: mit dem Überdrusse am Leben, mit der Ermüdung, mit dem Wunsche nach dem ,Ende'). (Nietzsche GM KSA 5: 366)

Die lebensbedrohliche Gefahr, die für Nietzsche von dieser Lebensform ausgeht, ist die, dass sich in diesem Sonderfall von Leben das Leben gegen sich selbst zu wenden beginnt, in dem es Wertschätzungen von sich kreiert, die ihm selbst nicht förderlich, sondern todfeind sind.

„Dies ist Alles im höchsten Grade paradox: wir stehen hier vor einer Zwiespältigkeit, die sich selbst zwiespältig will, welche sich selbst in diesem Leiden geniesst und in dem Maasse sogar immer selbstgewisser und triumphierender wird, als ihre eigene Voraussetzung, die physiologische Lebensfähigkeit, abnimmt." (Nietzsche GM KSA 5: 363)

Im Gegensatz zu anderen Lebensformen, denen an der Vermehrung von Freude und Lebendigkeit gelegen ist, lebt diese Figur gerade von der Reduktion der potentia agendi lebender Körper. Nicht die Vermehrung ihrer Lebenskraft und Wirkfähigkeit ist das Lebenselixier, in dem sie am besten gedeiht; sie findet vielmehr gerade dort ihre günstigsten Bedingungen vor, wo das Leben in Menschen schwindet. (Vgl. Nietzsche GM KSA 5: 339, 4I2) Irgendein selbstdestruktiver Todestrieb lässt diese Figur offenkundig die Selbstzerstörung des Lebens insgesamt anstreben und jene dunkle Alchemie vollziehen, die schon Spinoza

1 „Man komme mir nicht mit der Wissenschaft, wenn ich nach dem natürlichen Antagonisten des asketischen Ideals suche, wenn ich frage: ,wo ist der gegnerische Wille, in dem sich sein gegnerisches Ideal ausdrückt? ““ (Nietzsche GM KSA 5: 402) 
im Vorwort zu seinem Theologisch-politischen Traktat als geheime Basis tyrannischer Systeme beschrieben hat, wenn er schreibt:

„,Das große Geheimnis des monarchischen Systems und sein Hauptinteresse bestehen darin, die Menschen zu täuschen, indem sie die Furcht, mit der man sie im Zaum halten will, als Religion verkleiden; so dass sie für ihre Knechtschaft kämpfen, als handle es sich um ihr Heil.““ (Zit. nach Deleuze I988: 37; vgl. Spinoza 20I2: 6)

Gerade so, wie ein Tyrann die Unlust der Seelen braucht, um erfolgreich zu sein, ebenso brauchen trübselige Gemüter zu ihrer Erhaltung und Ausbreitung einen Tyrannen, um sich mit ihm an der Trübsal ihrer Leben zu rächen (vgl. Deleuze I988: 37 ).

Worin aber kann das Interesse des Lebens selbst an einer solchen Lebensform liegen? Was will ein Wille, der sich gegen sich selbst wendet? Der sich selbst Todfeind geworden ist? Irgendein Interesse des Lebens muss es an diesem Typus von Lebewesen doch geben, das ihn über alle Kulturen und Stände hinweg regelmäßig zum Leben erweckt? „Erwägen wir doch, wie regelmäßig, wie allgemein, wie fast $\mathrm{zu}$ allen Zeiten der asketische Priester in die Erscheinung tritt; er gehört keiner einzelnen Rasse an; er gedeiht überall; er wächst aus allen Ständen heraus." (Nietzsche GM KSA 5: 362)

Kann die Widernatur des Lebens, in der sich das Leben selbst Todfeind wird, selbst noch zur Definition von Leben gehören? Ist der real existierende Selbstwiderspruch, Leben gegen Leben,

„[...] physiologisch und nicht mehr psychologisch nachgerechnet, [doch] einfach Unsinn. Er kann nur scheinbar sein; er muss eine Art vorläufigen Ausdrucks, eine Auslegung, Formel, Zurechtmachung, ein psychologisches Missverständnis von Etwas sein, dessen eigentliche Natur lange nicht verstanden, lange nicht an sich bezeichnet werden konnte - ein blosses Wort, eingeklemmt in eine alte Lücke der menschlichen Erkenntnis.“ (Nietzsche GM KSA 5: 365)

Hier taucht sie also wieder auf, die Forschungslücke der traditionellen Philosophie, insofern sie in der Regel den schlafwandlerisch operierenden Appetit der Körper, samt ihren selbstdestruktiven Möglichkeiten, übersieht. Wenn es nämlich wahr ist, dass jedes Tier, auch in „das krankes Thier“ (Nietzsche GM KSA 5: 367), insgeheim nach dem Optimum der Bedingungen Ausschau hält, von denen es sich sein höchstes Glück verspricht, dann kann das Ziel des Wol- 
lens dieser selbstdestruktiven Lebensform nur in der Auslöschung des Lebens überhaupt liegen. Um lieber noch das Nichts zu wollen, als nicht zu wollen, muss für Nietzsche in der Leibes-Tiefe solcher Menschen in der Tat „ein Ressentiment sonder Gleichen“ herrschen: „das eines ungesättigten Instinktes und Machtwillens, der Herr werden möchte, nicht über Etwas am Leben, sondern über das Leben selbst, über dessen tiefste, stärkste, unterste Bedingungen; [...]“ (Nietzsche GM KSA 5:363).

\section{Spinozas Analyse des Selbstmörders}

Das Paradox der Selbstzerstörung des Lebens, das Nietzsche unter der Frage behandelt hat, wie die scheinbare Negation des Lebens in der Figur des asketischen Priesters zu denken ist, wird von Spinoza in Zusammenhang mit der Figur von Selbstmördern verhandelt. Auch für ihn weisen diese Menschen nämlich eine durch und durch paradoxe Begehrensstruktur auf, die uns vor ein Rätsel stellt. „Für Spinoza gehören Selbstzerstörer zu der gleichen Kategorie wie viereckige Kreise - sie sind ein chimärisches Nichts mit einer widersprüchlichen Essenz.“ (Cook 2006, I68)

Dass dem so ist, liegt an seiner Auffassung von Streben, der gemäß jedes Ding, insofern es in sich ist, notwendigerweise danach verlangt, in seinem Sein zu verharren. Kraft der von Spinoza angenommenen Strebenseinheit von Sein und Begehren ist es für ihn ganz und gar unverständlich, wie es möglich sein soll, dass ein Modi des Lebens von sich aus danach streben kann, sich das Leben zu nehmen. Denn dass „ein Mensch aus der Notwendigkeit seiner eigenen Natur danach strebt, nicht zu existieren oder sich in eine andere Gestalt zu verwandeln, ist so unmöglich, wie dass etwas aus nichts kommen könnte, wie jeder mit nur geringem Nachdenken sehen kann." (Spinoza 2007 [4p2Os]: 4I5; vgl. Cook 2006: I68)

Um diesen Selbstwiderspruch von Sein und Begehren auszuräumen, war Spinoza gezwungen, zwischen dem Streben eines aktual existierenden Körpers zu unterscheiden, das ihm selbst immanent ist, und der äußeren Gewalt, die er von anderen Körpern erleidet. Denn nur Körper, die von äußeren Umständen von ihrem eigenen Begehren gewaltsam so entfremdet worden sind, dass es ihnen verunmöglicht wird, ihr selbstimmanentes Begehren weiterhin zu äußern, befinden sich für Spinoza in einer so widrigen Lebenslage, dass es denkbar wird, dass sie ihr Nichtsein ihrem Sein vorziehen. Gilt doch, wie Lehrsatz 4 im dritten Teil seiner Ethik zeigt, dass kein Ding anders als von äußeren Ursachen 
zerstört und zur Negation seines eigenen Lebens bewegt werden kann (vgl. Spinoza 2007 [3p4]: 237).

So wie die Figur des asketischen Priesters für Nietzsche einen unerklärlichen Krieg gegen die natürlichsten Instinkte des Lebens führt, indem sie die Verneinung des Lebens betreiben, so erleidet jeder Selbstmörder für Spinoza seinen Tod kraft der Unterwerfung seines Lebenswillens unter äußere Formen der Knechtschaft, die ihn schlussendlich dazu bringen, sein Dasein zu verneinen, insofern es selbst unfähig geworden ist, seinen Lebenswillen unter den gegebenen Umständen weiterhin äußern zu können.

„Der Suizid (sofern er nicht wie Seneca als die Vermeidung des geringeren Übels von zwei Übeln erörtert wird, vgl. 4p2os) macht [für Spinoza] keinen Sinn und ist genau besehen nicht das Resultat einer freien Entscheidung, sondern Untat (im doppelten Sinne von Passivität und Laster) der Verzweiflung, die uns durch Zwang oder äußere Umstände auferlegt wird." (Wolf 2006: 202)

Wo immer sich ein Lebewesen sein Leben nimmt, dort handelt es für Spinoza ohnmächtigen Gemüts; überwältigt von äußeren Ursachen, die seiner eigenen Natur notwendigerweise widerstreben (vgl. Spinoza 2007 [4PI8s]: 4II). Und so kommt Spinoza zu folgendem Schluss:

„Niemand, sage ich, verschmäht Nahrung oder nimmt sich das Leben aus der Notwendigkeit seiner eigenen Natur, nur die tun es, die von äußeren Ursachen dazu gezwungen werden, was auf viele Weise geschehen kann. Einer mag sich selbst töten, weil er von einem anderen dazu gezwungen wird, [...] ein anderer, weil er, wie Seneca auf Befehl eines Tyrannen gezwungen wird, sich die Adern zu öffnen [...].“ (Spinoza 2007 [4p20s]: 4I5)

Bei all diesen Fällen wird man für Spinoza wider das eigene Begehren zwänglich zum Selbstmord getriebenen. Das zeigt sich besonders an der Tatsache, dass ein Wesen das Leben wieder bejahen würde, sobald es die knechtischen Verhältnisse losgeworden ist, die es zur Negation des eigenen Lebens drängten. „Denn ein jeder handhabt alles von seiner Affektivität her; [...] (Spinoza 2007 [3p2s]: 233). Und so besteht die ethische Aufgabe für Spinoza in Bezug auf diese Menschen darin, die Herzen all jener wieder zu befreien, die durch äußere knechtische Verhältnisse daran gehindert werden, ihrer eigenen Strebensnatur Ausdruck zu verleihen - damit es auch für sie wieder gute Gründe gibt, danach zu trachten, sich selbst im eigenen Dasein inmitten der Welt am Sein zu erhalten. 


\section{Denken in affectus}

Um von der hegemonialen Vormachtstellung eines Denkens loszukommen, das vornehmlich gegen den Affekt, und nicht „in affectus“ (Spinoza 2007: 562) denkt, muss man in Bezug auf das gängige Bild des Denkens offenkundig radikal umlernen, indem man mit Nietzsche erkennt, dass „man noch den größten Theil des bewussten Denkens unter die Instinkt-Thätigkeiten rechnen muss, sogar im Falle des philosophischen Denkens;“, denn „das meiste bewusste Denken eines Philosophen ist durch seine Instinkte heimlich geführt und in bestimmte Bahnen gezwungen." (Nietzsche JGB KSA 5: I7)

Für jene, die mit ihm gelernt haben, Philosophen bei der Bestimmung ihres höchsten Guts zwischen die Zeilen und auf die Finger zu schauen, liegt offen zu Tage, dass auch den rationalen Überlegungen der Philosophen und ihrer systematischen Suche nach Vernunftgründen ein Subtext libidinöser Erwägungen zugrunde liegt, die sie instinktiv dazu treiben, dem dominanten Instinkt ihres Begehrens denkerisch zum Ausdruck zu verhelfen.

Um ein wirkliches Gegenideal gegen asketische Wertschätzungen entstehen zu lassen, braucht es für Nietzsche daher, wie er in Jenseits von Gut und Böse schreibt, eine neue Gattung von Philosophen, die definitiv „irgend welchen anderen umgekehrten Geschmack und Hang haben als die bisherigen [...]" (Nietzsche JGB KSA 5: I7). Künstlerphilosoph_innen, denen im Unterschied zu ihren Ahnen nicht mehr die Verwerfung der Sinnlichkeit, sondern ihre Nobilitierung am Herzen liegt und die daher bereit sind, die obskuren Zonen ihrer oft schlafwandlerisch operierenden Körper aufzusuchen und zu kultivieren. Dieses neue Geschlecht von Künstlerphilosoph_innen, das Nietzsche, wie er in Jenseits von Gut und Böse schreibt, schon kommen sieht, wird das Denken definitiv nicht mehr als Befreiung von der Sinnlichkeit definieren, sondern als Form der Intensivierung und leiblichen Nobilitierung unseres Begehrens.

In seinem wunderbaren Text Die absolute Immanenz betont Giorgio Agamben, dass schon Spinoza die Erfahrung wirklicher Freiheit als Möglichkeit der faktischen Äußerung der selbstimmanenten Natur unseres Begehrens dachte. (Vgl. Agamben I998: 77-I27; Deleuze I993: 269-283; Hampe 2006: 260-26r; Spinoza 2007 [5p42]: 593-595). Frei ist ein lebendiger Körper folglich genau dann, wenn er sich in einem Milieu befindet, das ihm die Äußerung seines selbstimmanenten Begehrens erlaubt. Da einem solchen Körper sein Da-sein notwendigerweise begehrenswert erscheinen wird, wird er auch danach streben, sich selbst am Sein zu erhalten. Was nichts anderes heißt: „Im conatus fallen folglich Begehren und Sein restlos zusammen." (Agamben I998: II9) 
Leben heißt für Spinoza sein - und zwar im Sinne des aktuellen Daseins eines wirklich existierenden Körpers. Wirklich lebendig und frei ist ein real existierender Körper für ihn aber immer erst dort, wo er sich in seinem weltweiten Dasein so in sich selbst befindet, dass ihm sein Dasein zum Ausdrucksgeschehen seines weltweiten Begehrens wird. Wo solches geschieht ist ein Körper notwendigerweise selig. Denn: „Bei Spinoza ist die Idee der Glückseligkeit daran gebunden, sich selbst als immanente Ursache zu erfahren. Diese Erfahrung nennt er acquiescentia in se ipso [Selbstzufriedenheit] und definiert sie genau als laetitia, concomitante idea sui tamquam causa" (Agamben I998, I23); als Lust, verbunden mit der Idee seiner selbst als Ursache.

Agamben hat darauf hingewiesen, dass es sich bei der Formulierung acquiescentia in se ipso um einen Neologismus von Spinoza handelt, den kein lateinisches Wörterbuch verzeichnet, so dass es sich um einen Hebraismus (oder Ladinismus) handelt, „der gebildet wurde, um den Kulminationspunkt der Bewegung der immanenten Ursache auszudrücken. Und Deleuze benutzt den Begriff ,Glückseligkeit' in eben diesem Sinne, als die wesentlichste Eigenschaft, die einem Leben... zukommt: die beatitudo ist die Bewegung der absoluten Immanenz." (Agamben 1998: 124-I25)

Selig ist ein Leben, das sein eigenes Begehren begehren kann. Wo eine solche Bejahung des eigenen Begehrens Ereignis wird, vollzieht ein Leben die ewige Selbstbejahung des Lebens selbst durch sich selbst und in sich selbst: Die Bewegung absoluter Immanenz...

\section{Bibliographie}

Agamben, Giorigo: Bartleby oder die Kontingenz gefolgt von Die absolute Immanenz, Berlin: Merve Verlag 1998.

Böhler, Arno: Singularitäten. Vom zu-reichenden Grund der Zeit. Vorspiel einer Philosophie der Freundschaft, Wien: Passagen Verlag 2005.

Böhler, Arno/Granzer Susanne: "FALLING Prey to Sleep", in: Scores \#6, On Addressing, Jg. 5/20I4, Heft 6, Wien: Tanzquartier Wien.

Butler, Judith/Laclau, Ernesto/Slavoj Žižek: Kontingenz, Hegemonie, Universalität. Aktuelle Debatten zur Linken [2000, 20II], übers. u. hg. v. Gerald Posselt u. Mitar. v. Sergej Seitz, Wien-Berlin: Verlag Turia + Kant 2013. 
Cook, Thomas: „Der Conatus: Dreh- und Angelpunkt der Ethik“, in: Michael Hampe/Robert Schnepf: Baruch de Spinoza. Ethik in geometrischer Ordnung dargestellt, Berlin: Akademie Verlag 2006, S. I5I-I70.

Damasio, R. Antonio: Der Spinoza-Effekt. Wie Gefühle unser Leben bestimmen, übers. v. Hainer Kober, Berlin: List Verlag 2009 (5. Auflage).

Deleuze, Gilles: Differenz und Wiederholung [I968], übers. v. Joseph Vogl, München: Wilhelm Fink Verlag 1997 (2. Auflage).

Deleuze, Gilles: Spinoza. Praktische Philosophie [I98I], übers. v. Hedwig Linden, Berlin: Merve Verlag I988.

Deleuze, Gilles: Spinoza und das Problem des Ausdrucks in der Philosophie [I968], übers. v. U. J. Schneider. München: Wilhelm Fink Verlag I993.

Derrida, Jacques: Marx' Gespenster, übers. v. Susanne Lüdemann, Frankfurt a. M.: Fischer Taschenbuch I995.

Hampe, Michael: „Das vernunftgeleitete Leben freier Menschen“, in: ders./Robert Schnepf: Baruch de Spinoza. Ethik in geometrischer Ordnung dargestellt, Berlin: Akademie Verlag 2006, S. 25I-265.

Heidegger, Martin: Kant und das Problem der Metaphysik, Gesamtausgabe Band 3, hg. v. Friedrich-Wilhelm von Herrmann, Frankfurt a. M.: Vittorio Klostermann i99ı.

Husserl, Edmund: Analysen zur passiven Synthesis [I9I8-I926], Husserliana XI, The Haag: Martinus Nijhoff ig66.

Matheron, Alexandre: „Bemerkungen zur Unsterblichkeit der Seele bei Spinoza“, in: Michael Hampe/Robert Schnepf: Baruch de Spinoza. Ethik in geometrischer Ordnung dargestellt, Berlin: Akademie Verlag 2006, S. 297-307.

Nietzsche, Friedrich: Sämtliche Werke. Kritische Studienausgabe, hg. v. Giorgio Colli und Mazzimo Montinari, München/Berlin/New York: DTV Walter de Gruyter I967-I977

Genealogie der Moral, Band 5, S. 245-4I2.

Jenseits von Gut und Böse, Band 5, S. 9-243.

Götzen-Dämmerung, Band 6, S. 55-г6г.

Rölli, Marc: Philosophie des Transzendentalen Empirismus, Wien: Verlag Turia + Kant 2003 .

Schopenhauer, Arthur: Die Welt als Wille und Vorstellung, Bd. I, Sämtliche Werke in 6 Bänden, Stuttgart: Reclam Verlag I892 (2. Auflage).

Spinoza: Ethik in geometrischer Ordnung dargestellt, lat.-dt., übers. u. hg. v. Wolfgang Bartuschat, Hamburg: Felix Meiner Verlag 2007.

Spinoza: Theologisch-politischer Traktatus, übers. und hg. v. Wolfgang Bartuschat, Hamburg: Felix Meiner Verlag 2012. 
Wolf, Jean-Claude: „Menschliche Unfreiheit und Desillusionierung (4praef4pI8)“, in: Michael Hampe/Robert Schnepf: Baruch de Spinoza. Ethik in geometrischer Ordnung dargestellt, Berlin: Akademie Verlag 2006, S. I97-2I4. 